\title{
Exchange Coupling in Magnetic Semiconductor Multilayers and Superlattices
}

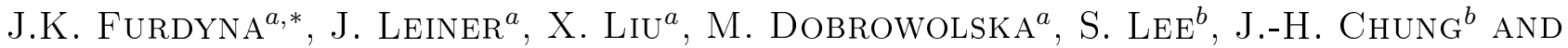 \\ B.J. KIRBY ${ }^{c}$ \\ ${ }^{a}$ Department of Physics, University of Notre Dame, Notre Dame, IN 46556, USA \\ ${ }^{b}$ Department of Physics, Korea University, Seoul 136-713, Korea \\ ${ }^{c}$ Center for Neutron Research, NIST, Gaithersburg, MD 20899, USA
}

\begin{abstract}
The study of ferromagnetic semiconductors continues to be of great interest because of their potential for spintronic devices. While there has been much progress in our understanding of ferromagnetic semiconductor materials - particularly of the canonical III-V system $\mathrm{Ga}_{1-x} \mathrm{Mn}_{x} \mathrm{As}$ - many issues still remain unresolved. One of these is the nature of interlayer exchange coupling in GaMnAs-based multilayers, an issue that is important from the point of view of possible spintronic applications. In this connection, it is important to establish under what conditions the interlayer exchange coupling between successive GaMnAs layers is antiferromagnetic or ferromagnetic, since manipulation of such interlayer exchange coupling can then be directly applied to achieve giant magnetoresistance and other devices based on this material. In this review we will describe magneto-transport, magnetization, and neutron reflectometry experiments applied to two types of GaMnAs-based multilayer structures - superlattices and tri-layers - consisting of GaMnAs layers separated by non-magnetic GaAs spacers. These measurements serve to identify conditions under which AFM coupling will occur in such GaMnAs/GaAs multilayer systems, thus providing us the information which can be used for manipulating magnetization (and thus also giant magnetoresistance) in structures based on the ferromagnetic semiconductor GaMnAs.
\end{abstract}

PACS: 75.50.Pp, 61.05.fj, 75.47.De, 75.70.Cn

\section{Introduction}

The study of dilute ferromagnetic semiconductors (FMSs) continues to be of intense interest because of their potential for spin-electronic device applications [1]. There has been much progress in our understanding of FMS materials, particularly of the canonical III-V system $\mathrm{Ga}_{1-x} \mathrm{Mn}_{x} \mathrm{As}$ [2].

In III-V-based FMSs, $\mathrm{Mn}^{2+}$ ions replace group-III cations, thus providing magnetic moments, and also acting as acceptors. It is of key importance that the holes arising from the presence of the $\mathrm{Mn}^{2+}$ acceptors then mediate interactions between magnetic moments localized on the Mn ions, thus leading to ferromagnetic order in these III-V-based FMSs with a relatively high Curie temperature, which currently approaches $190 \mathrm{~K}[3,4]$. Even though this Curie temperature does not allow room temperature applications, GaMnAs can already serve as a model material for testing proof-of-concept spintronic devices.

Many of the spintronic devices under consideration (e.g., spin valves) involve FMS/non-FMS multilayers of

\footnotetext{
* corresponding author; e-mail: furdyna@nd.edu
}

various forms. Theoretical models predict both ferromagnetic (FM) and antiferromagnetic (AFM) interactions between the FMS layers, the type of inter-layer exchange coupling (IEC) being of course crucial to the device operation [5-8]. It has been shown in a number of studies that the type of IEC - whether it is FM or AFM - depends sensitively on the thickness and doping of the non-FM spacers between the FM layers [9, 10]. Until recently, however, only FM coupling had been observed in GaMnAs/GaAs and GaMnAs/AlGaAs multilayers [11-15].

As an illustration, Fig. 1 shows polarized neutron reflectometry (PNR) measurements taken on GaMnAs/ GaAs superlattices, demonstrating FM coupling between the magnetic layers of the structure.

The main motivation for the present work is to achieve and further understand AFM interactions between neighboring magnetic layers, since AFM IEC can enable us to obtain structures manifesting giant magnetoresistance (so-called "GMR"), which constitutes the basis for important devices. In such structures the application of a small external magnetic field (or, as will be argued, other external stimuli) can reverse magnetization of magnetic layers from AFM to FM, resulting in drastic changes of resistance. In the case of GaMnAs/GaAs multilayers being 


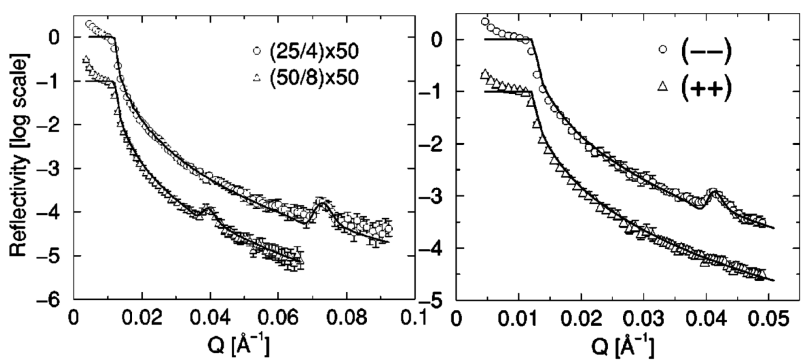

Fig. 1. (left) Unpolarized neutron reflectivity profiles for $(25 \mathrm{ML} / 4 \mathrm{ML}) \times 50$ and $(50 \mathrm{ML} / 8 \mathrm{ML}) \times 50$ GaMnAs/GaAs superlattices. (right) Polarized neutron reflectivity profiles for the $(50 \mathrm{ML} / 6 \mathrm{ML}) \times 50 \mathrm{GaMnAs} /$ GaAs superlattice taken in $2 \mathrm{G}$ magnetic field. The $(++)$ and $(--)$ represent two non-spin flip (NSF) reflectivity channels for the neutron spins parallel and antiparallel, respectively, to the applied field. The data for spin-up non-spin flip (denoted $(++)$ ) scattering is shifted down for clarity. Reprinted with permission from Phys. Rev. B, Ref. [13]. C(2001) by the American Physical Society.

considered here, such coupling depends on the doping of the non-magnetic layer separating the magnetic GaMnAs layers, thus allowing us to study the role which charge carriers play in exchange coupling between magnetic layers. Besides the interesting physics of this effect, this also enables (via injection of carriers into the spacer layers) the reversal of magnetization in the GaMnAs layers from AFM to FM and vice versa.

In this review we will focus on the features and recent discoveries of AFM interlayer coupling in GaMnAs/ $\mathrm{GaAs} / \mathrm{GaMnAs}$ superlattices and trilayers, with emphasis on the effect of carriers in spacer layers on the process of IEC between the magnetic layers of the composite structure.

\section{Observation of AFM IEC in GaMnAs/GaAs superlattices}

\subsection{Magnetization results observed on GaMnAs/GaAs superlattices}

To study the interlayer exchange coupling phenomena, we investigated two GaMnAs/GaAs superlattices (SLs) consisting of ten ferromagnetic $\mathrm{Ga}_{0.97} \mathrm{Mn}_{0.03}$ As layers separated by nonmagnetic GaAs layers. The two SLs are structurally identical (i.e., they have the same SL period of $d_{\mathrm{SL}} \approx 26 \mathrm{ML}$ ), but one in one of the SLs the GaAs spacers are intentionally Be-doped, and remain undoped in the other.

Figure 2 shows the magnetization curves observed in a series of DC fields applied along the [100] direction, which is approximately parallel to the magnetic easy axis at the lowest temperature. In the sample with undoped spacers, the magnetization increases below $T \approx 60 \mathrm{~K}$ following the typical behavior of ferromagnetic GaMnAs. In sharp contrast, in the sample with Be-doped spacers the temperature behavior of magnetization measured in low fields is

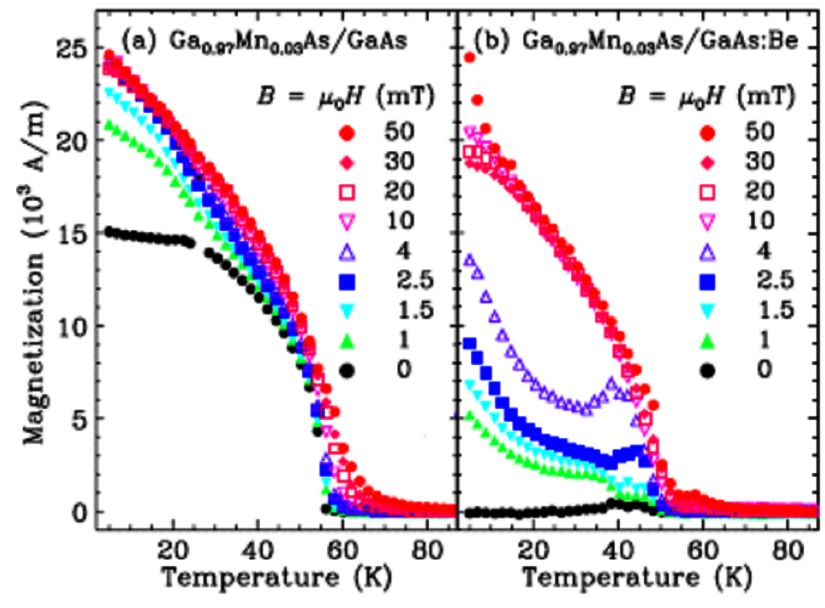

Fig. 2. Temperature dependence of magnetization of (a) $\mathrm{Ga}_{0.97} \mathrm{Mn}_{0.03} \mathrm{As} / \mathrm{GaAs}$ and (b) $\mathrm{Ga}_{0.97} \mathrm{Mn}_{0.03} \mathrm{As} /$ GaAs:Be superlattices. The data were collected while cooling, with the magnetic field applied along the [100] direction.

very different. In particular, at zero field, the net magnetization is almost completely suppressed, showing only a very weak signal below $T_{\mathrm{C}}$. Such a large decrease in net magnetization indicates significant changes in exchange coupling due to Be doping, strongly suggesting that IEC between ferromagnetic layers is antiferromagnetic. At higher fields the magnetization reflects the combined effects of IEC and the applied external field, whose relative strengths vary as the temperature changes. For example, the magnetization measured at $4 \mathrm{mT}$ rises around $50 \mathrm{~K}$, then drops as the temperature is lowered to below $40 \mathrm{~K}$, followed by another upturn; and as the field is further increased (see, e.g., data for $10 \mathrm{mT}$ and higher), the magnetization of the superlattice eventually acquires "normal" FM behavior, suggesting that these higher fields are now sufficient to overcome the effect of IEC, resulting in ferromagnetic spin alignment in all layers of the SL.

\subsection{Polarized neutron reflectometry measurements on GaMnAs/GaAs superlattices}

Although the temperature behavior of the magnetization suggests that interlayer coupling is FM for the undoped SL and AFM for the Be-doped specimen, this cannot be definitively concluded based on magnetization measurement alone. Polarized neutron reflectometry (PNR), however, is an ideal method to investigate spin alignment of the superlattices, thus establishing types of interlayer coupling between the magnetic layers. We have therefore performed extensive PNR measurements on structures of interest in this paper, as described below. We note parenthetically that, in addition to its sensitivity to the relative spin arrangement in the magnetic layers, polarized as well as unpolarized neutron reflectivity is also sensitive to the sample structure.

The unpolarized neutron reflectivity measured on the Be-doped sample above $T_{\mathrm{C}}$ (in this case at $100 \mathrm{~K}$, where 


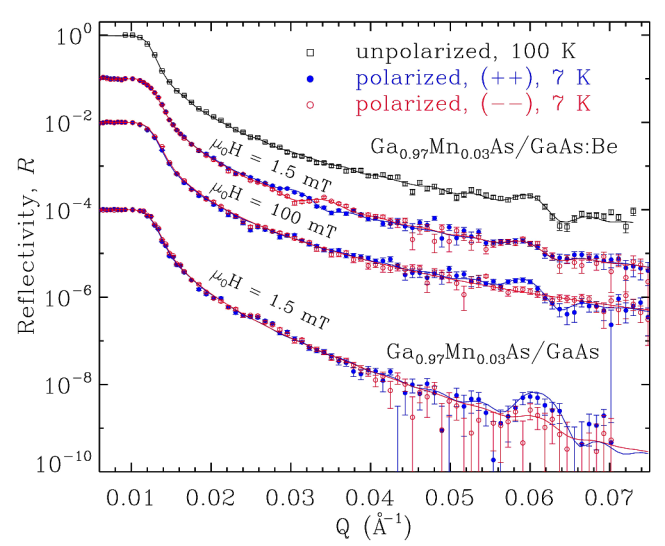

Fig. 3. Unpolarized and polarized neutron reflectivities observed on SLs with Be-doped and undoped spacer layers with an external field applied along the [110] direction. The curves are shifted vertically for clarity. The solid lines are fits to the data using the models described in the text.

the GaMnAs layers are in the paramagnetic phase) is plotted in the uppermost part of Fig. 3, showing a Bragg peak at $Q=0.062 \AA^{-1}$, which arises from the superlattice structure and corresponds to a periodicity of $d_{\mathrm{SL}}=104 \AA$. The S-shaped profile of the Bragg peak (instead of a simple peak) is attributed to the presence of a capping layer, a feature that is reproduced by model fitting [16].

To identify the spin alignment between the magnetic layers in the structure, we performed neutron reflectivity measurements below $T_{\mathrm{C}}$ using a polarized neutron beam. In this polarization-selective experiment we will focus only on the reflection intensities for the non-spin-flip (NSF) process $((++)$ and $(--))$, since the reflectivity intensities for the spin-flip (SF) case $((+-)$ and $(-+))$ are much weaker (by several orders of magnitude) for the FMS layers. The data shown in the second-from-the-top curve in Fig. 3 represents the two NSF reflectivities obtained at $7 \mathrm{~K}$ in an external field of $1.5 \mathrm{mT}$ applied along the [110] direction during the measurement. The NSF-NPR data show an additional peak at $Q=0.031 \AA^{-1}$ which is split for the two neutron spin polarizations, while the structural Bragg peak is nearly unchanged. The new spin-split peak provides a signature that there is an additional periodicity corresponding to twice the structural SL period, caused by the spin alignments in the SL parallel or antiparallel to the incident neutron polarization. It is evident that such magnetic periodicity is consistent with antiparallel alignment between the successive FMS layers, and clearly indicates the presence of spontaneous AFM IEC in SL with Be-doping.

Let us note, however, that this splitting of peaks at $Q=0.031 \AA^{-1}$ is fully suppressed when the applied field is increased to $100 \mathrm{mT}$, as is seen in the third-from-the-top curve in Fig. 3. At the same time a new splitting is now observed at the structural Bragg peak. Since the structural period of the SL is identical to the magnetic period of the SL when the layers are FM-coupled, this peak is a superposition of the structural and magnetic components, the splitting between the two spin-polarized neutron beams arising because the spins in the FMS layers of the superlattice are either parallel or antiparallel to the spin polarization of the beam. We have repeated the PNR measurement under the same condition (i.e., at $7 \mathrm{~K}$ and an external field of $1.5 \mathrm{mT}$ ) on the undoped sample. The data are shown in the fourth curve from the top in Fig. 3. In contrast to the case of the Be-doped SL, the undoped SL shows a splitting of only its structural Bragg peak, arising from the superposition of the structural and magnetic Bragg reflections, as discussed above. This indicates that the FMS layers in the undoped SL are aligned ferromagnetically along the applied field, thus leading to the conclusion that IEC in the SL with undoped spacer layers is very different from the case when the spacers are doped with Be.

\subsection{Magnetotransport observed on GaMnAs/GaAs superlattices}

As already mentioned, the AFM IEC in a magnetic multilayer is responsible for the giant magnetoresistance (GMR) effect observed in such multilayers in magnetotransport. Figure 4 shows the magnetoresistance (MR) data measured at $30 \mathrm{~K}$ with the magnetic field applied near the [110] direction for both Be-doped and undoped SLs. The SL with undoped spacers (lower part) shows an anisotropic magnetoresistance (AMR) that is determined only by the angle between the magnetization vector and the direction of the current. Such AMR is typical of ferromagnetic GaMnAs, and is normally observed in single layer films as well $[17,18]$. From this we infer that the IEC between the ferromagnetic $\mathrm{Ga}_{0.97} \mathrm{Mn}_{0.03}$ As layers in the SL with undoped spacers layers is most likely FM, and the SL acts magnetically as a single layer. This is completely consistent with the finding from the magnetization and PNR experiments.

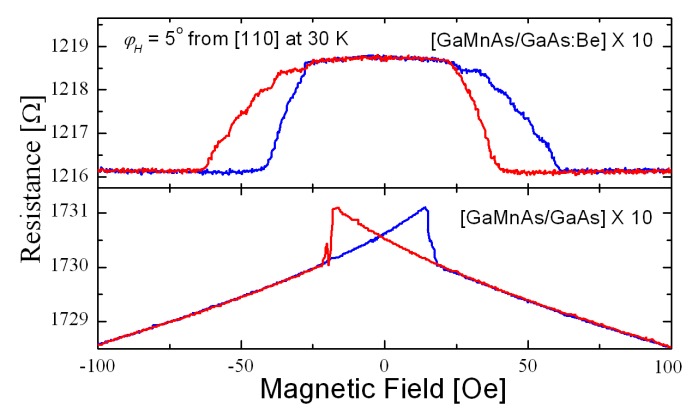

Fig. 4. Magnetoresistance data for GaMnAs/GaAs superlattices with doped and undoped spacer layers.

In the case of the SL with Be-doped spacers, the resistance (see upper part of Fig. 4) shows a relatively flat maximum at zero field, that persists over a finite field 
range (in this case up to $B \approx 25 \mathrm{Oe}$ ). Then the resistance makes a steep transition with further increasing the field above $B \approx 66 \mathrm{Oe}^{*}$. A hysteresis is observed on the return path (i.e., on decreasing the field), because the reverse transition occurs at lower fields. Nevertheless, the zero-field resistance is fully recovered at $B \approx 25$ Oe. When the reverse field is applied, the observed magnetoresistance (MR) is exactly symmetric about zero. Such spontaneous recovery of the zero-field resistivity strongly suggests that there is a spontaneous coupling between the magnetic layers. The presence of the AFM IEC in the Be-doped sample has already been confirmed in the polarized neutron reflectivity discussed above [19]. Thus we conclude that the effect observed in the present magnetotransport measurements is a GMR-like effect qualitatively similar (but not quantitatively, since the change in resistance is not large) to that often observed in metallic ferromagnetic multilayers in which AFM IEC is present [20-22].

\section{Observation of AFM IEC in GaMnAs/GaAs/ GaMnAs trilayers}

The data obtained on superlattices show that interlayer coupling occurs in the GaMnAs/GaAs multilayer combination, and that it appears when certain concentrations of free carriers are present in the spacer (GaAs) layers. Using this as a starting point, a study of two GaMnAs layers separated by a non-magnetic GaAs spacer is particularly useful, since such a trilayer enables one to "zoom in" on the specific properties of GaMnAs that determine the IEC. The understanding of such trilayer properties is also important because this geometry constitutes a prototype structure which can be exploited in device (e.g., GMR) applications.

\subsection{Magnetization results observed on GaMnAs/GaAs/GaMnAs trilayers}

\subsubsection{Temperature dependence of magnetization}

SQUID measurements were used to study the temperature dependence of the trilayer magnetization $M(T)$. Magnetization data for a trilayer with a $4.3 \mathrm{~nm}$ spacer and an average hole concentration over the entire trilayer of $2 \times 10^{20} \mathrm{~cm}^{-3}$ are shown in Fig. 5. The sample was oriented so that the measured magnetization and the applied field $\boldsymbol{H}$ were parallel to the uniaxial easy axis [110] of the GaMnAs layers [23]. The sample was either field-cooled (FC) in a field of $1.5 \mathrm{mT}$, or zero-field-cooled (ZFC), and the magnetization was then measured as the temperature was increased in zero field.

As shown in Fig. 5a, three distinct behaviors are observed in three different temperature regimes. Below

\footnotetext{
* The reader will notice also weak steps on the "slope" of the MR curve, seen particularly clearly in curves collected as the field increases. These steps correspond to successive switching of magnetization in individual layers of the SL. This effect will be discussed in a future publication.
}

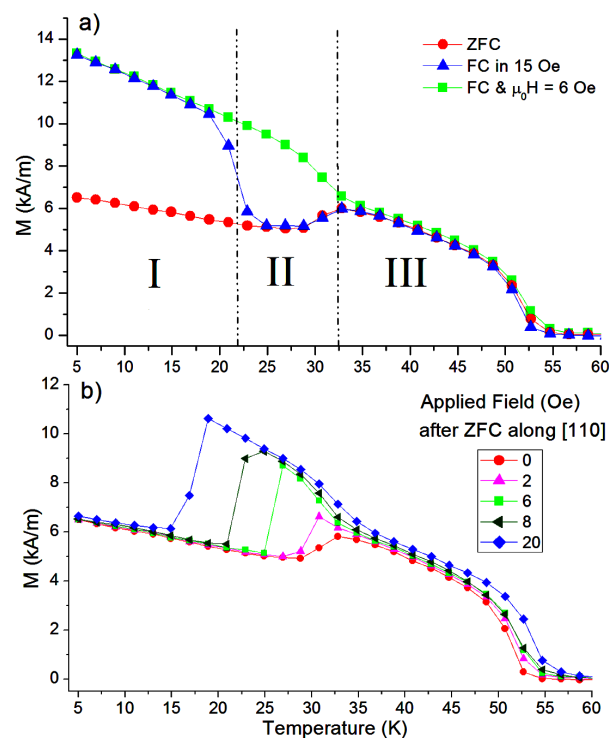

Fig. 5. Temperature dependence of remnant magnetization in a trilayer sample with respect to the [110] crystallographic direction. (a) Data collected while warming after cooling from $200 \mathrm{~K}$ to $5 \mathrm{~K}$ in either zero field or $1.5 \mathrm{mT}$. Also shown are $\mathrm{FC}$ data measured in an applied field of $0.6 \mathrm{mT}$. (b) Data collected while warming in the indicated applied fields, after cooling from $200 \mathrm{~K}$ to $5 \mathrm{~K}$ in zero field.

$20 \mathrm{~K}$ the FC and ZFC data differ dramatically: field cooling results in a conspicuous enhancement of the magnetization, while a suppression of magnetization is observed in the ZFC data. We attribute these characteristics to a parallel alignment (addition) or an antiparallel alignment (subtraction) of the magnetization $M$ of the two GaMnAs layers. Moreover, a clear dip in $M(T)$ is observed for the FC data around $30 \mathrm{~K}$, with a minimum corresponding exactly to the $\mathrm{ZFC}$ value of $M$ at the same temperature, suggesting that the parallel alignment of $\boldsymbol{M}$ in the GaMnAs layers that occurred at low temperature in the FC case has switched to an antiparallel alignment as $T$ exceeded $\approx 20 \mathrm{~K}$. Thus the magnetization data show clear signs of AF IEC. Let us note, however, that when the temperature dependence of $\boldsymbol{M}$ is measured in a finite field (in this case $0.6 \mathrm{mT}$ ), the $\mathrm{FC}$ data does not show this dramatic dip, indicating that the presence of a small applied field prevents the AF reorientation from occurring. Finally, above $30 \mathrm{~K}$ the $M(T)$ curves for FC and ZFC are seen to merge, first increasing, and then decreasing as $T$ increases. In this temperature region the value of $M$ suggests that only one GaMnAs layer (bottom) contributes to $M(T)$, as discussed later in the paper.

Further exploring the temperature dependence of magnetization in the trilayer sample with a doped spacer, Fig. 5b shows the measured magnetizations as the temperature was increased in various applied fields after ZFC. This shows a clear trend: the higher the applied field after ZFC, the lower the temperature at which the magnetization undergoes a sharp transition to larger 
magnitudes, which corresponds to switching of magnetization in the top from an antiparallel to a parallel magnetic alignment with respect to the magnetization of the bottom layer. We also collected data for the complimentary scenario (not shown) where, after field cooling, there is a transition from parallel to antiparallel alignment of the two layers [24].

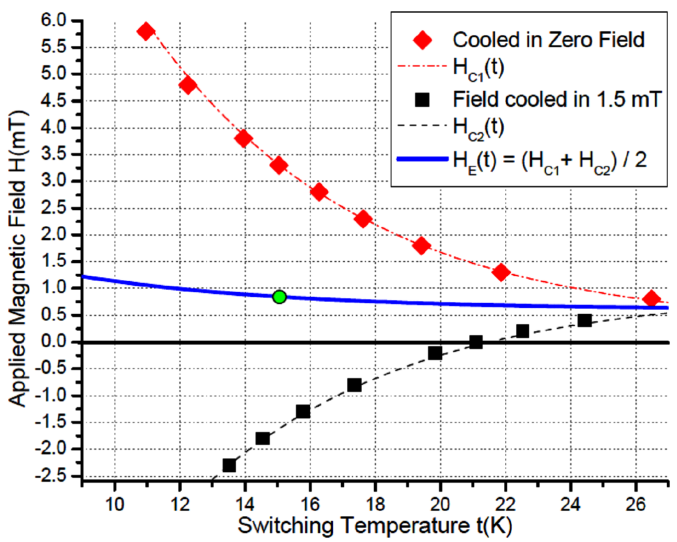

Fig. 6. Plot of magnetic fields at which the alignment changes from antiparallel to parallel as a function of temperature. The ZFC and FC data were each fitted to an exponential decay function with the same decay constant. The value of the exchange field $H_{\mathrm{E}}$ was then determined by taking the average of the two curves, shown as the solid blue line. The green point marks the value of $H_{\mathrm{E}}$ determined from a direct hysteresis loop measurement (Fig. 7).

The dependence of the IEC field $H_{\mathrm{E}}$ on temperature can be estimated by plotting the applied field $\boldsymbol{H}$ as a function of the temperature at which the parallel-to-antiparallel transition occurs (see Fig. 6). These data, representing the two coercive fields $H_{\mathrm{C} 1}$ and $H_{\mathrm{C} 2}$ as a function of $T$, can be fitted using exponential decay functions with the same decay constant. The average of $H_{\mathrm{C} 1}$ and $H_{\mathrm{C} 2}$ then gives the magnitude of the exchange field $H_{\mathrm{E}}$. This is equivalent to measuring the center of the minor hysteresis loop of top layer as a function of temperature. Also shown in Fig. 6 (by open circle) is the value of $H_{\mathrm{E}}$ estimated from the minor hysteresis loop at $15 \mathrm{~K}$ (as discussed in the next section), corroborating our method of estimating $H_{\mathrm{E}}$.

\subsubsection{Hysteresis loops of GaMnAs/GaAs/GaMnAs trilayer}

Further insight into IEC can be gained by examining the hysteresis of the GaMnAs/GaAs/GaMnAs trilayer at different temperatures shown in Fig. 7. The hysteresis curves show a two-step magnetization reversal both at $15 \mathrm{~K}$ and $30 \mathrm{~K}$. The fact that the top GaMnAs layer is half as thick as the bottom layer automatically allows us to attribute the smaller loop to the top layer. The $15 \mathrm{~K}$ loop shows a decreasing magnetization step only after the field in the return cycle has been swept past zero to $-2 \mathrm{mT}$. In contrast, the magnetization curve at
$30 \mathrm{~K}$ clearly shows the reversal of magnetization of the top GaMnAs layer occurring before the field reaches zero, indicating robust AF alignment that returns after cycling to saturation, corroborating what has already been seen in the temperature dependence data. Qualitatively we can picture this as the top GaMnAs layer "feeling" an exchange bias field $H_{\mathrm{E}}$ from the bottom layer, that acts to accelerate the reversal of magnetization even before the applied field reaches zero. Moreover, let us note that the minor hysteresis loop in Fig. $7 \mathrm{~b}$ is centered to the right of $H=0$, from which we can infer that at $30 \mathrm{~K}$ the value of $H_{\mathrm{E}}$ is $\approx 0.73 \mathrm{mT}$. A closer inspection of Fig. 7a shows that the center of the minor hysteresis loop for $15 \mathrm{~K}$ also lies to the right of $H=0$, shifted by $\approx 0.85 \mathrm{mT}$. This indicates that $H_{\mathrm{E}}$ is also present at that lower temperature - in fact it is stronger than at $30 \mathrm{~K}$ - but is less conspicuous because it is overshadowed by the much larger coercive field at the lower temperature.

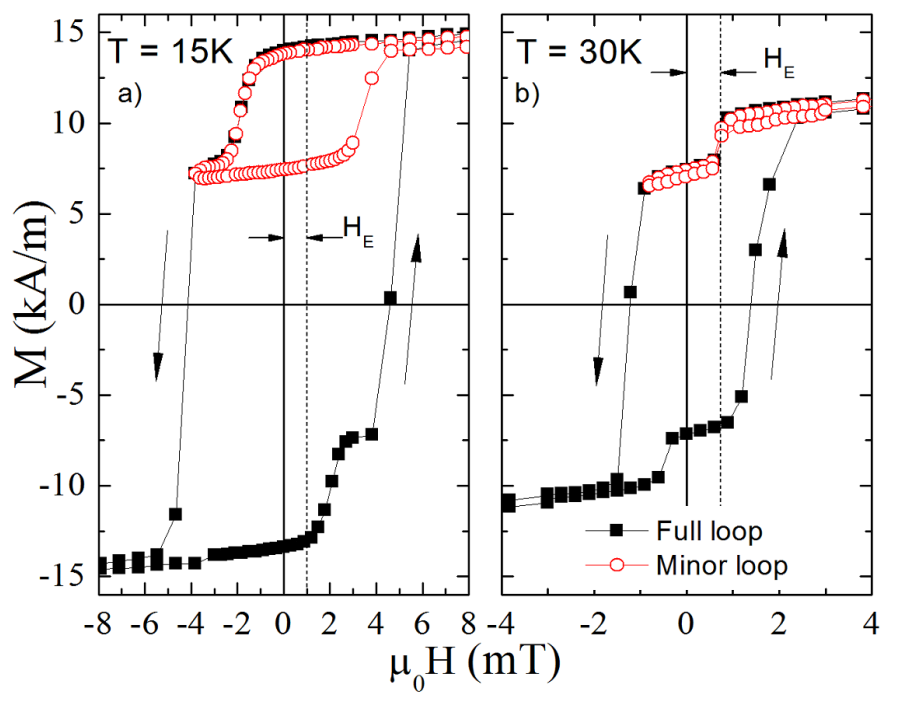

Fig. 7. Magnetization curves for field along the [110] direction taken at different temperatures. (a) $T=15 \mathrm{~K}$, and (b) $T=30 \mathrm{~K}$. Filled and empty symbols show the full and minor loops, respectively.

\subsection{Polarized neutron reflectometry on GaMnAs/GaAs/GaMnAs trilayers}

We now show evidence obtained by PNR for the picture of IEC presented above. PNR measurements were carried out for this sample with $\boldsymbol{H}$ applied along the [110] GaMnAs direction, using an incident monochromatic neutron beam (with neutron wavelength of $0.475 \mathrm{~nm}$ ) spin-polarized either parallel (spin-up) or antiparallel (spin-down) to the applied field $\boldsymbol{H}$. The spin-up and spin-down non spin-flip specular reflectivities measured as a function of the scattering wave vector $Q$ provide information on the depth profile of the in-plane magnetization parallel to $\boldsymbol{H}$, [25]. which can be model-fitted using exact dynamical calculations [26] to determine the 
actual magnetizations of the individual GaMnAs layers within the trilayer structure [10].

After cooling to either $5 \mathrm{~K}$ or $30 \mathrm{~K}$ in zero field, a small field $(<1 \mathrm{mT})$ was applied to ensure polarization of the neutron beam, and PNR spectra were measured to determine the spontaneous (i.e., ZFC) magnetization state of the trilayer. The field was then cycled between +800 and $-800 \mathrm{mT}$ before returning below $1.0 \mathrm{mT}$ in order to investigate the robustness of the initial magnetization state. Since the difference between the spin-up and spin-down non-spin-flip reflectivities is small, it is convenient to plot the fitted PNR data in the form of spin asymmetry (i.e., the difference between spin-up and spin-down reflectivities divided by their sum), as shown in Fig. 8 .

For this case, the most important feature of the spin asymmetry is the ratio of the magnitudes of the peak occurring at the lowest- $Q$ to that at the second-lowest $Q$, a quantity which we define as $\beta$. Model calculations [26] show that when $\beta>1$, the alignment of the two GaMnAs layers within the trilayer is parallel (FM), while $\beta \leq 1$ corresponds to antiparallel (AF) alignment. For the $5 \mathrm{~K}$ measurement in $0.6 \mathrm{mT}$ immediately after ZFC (Fig. 8a), the magnitude of the first peak is significantly smaller than that of the second, giving $\beta \approx 0.7$, thus indicating AF alignment of $M$ in the two GaMnAs layers. This conclusion is borne out by quantitative fitting (solid line), which yields $M_{\text {top }}=-18 \mathrm{kA} \mathrm{m}^{-1}$ for the top GaMnAs layer and $M_{\text {bot }}=+23 \mathrm{kA} \mathrm{m}^{-1}$ for the bottom layer (illustrated on the bottom of Fig. 8, which also shows the surface roughness of $\boldsymbol{M}$ ). The sensitivity of this fitting procedure is demonstrated by calculations with the magnetization of the top layer reversed (dashed curve), which strongly deviates from the data. After field cycling and returning to $0.6 \mathrm{mT}$ at $5 \mathrm{~K}$ (Fig. $8 \mathrm{~b}$ ), $\beta$ increases dramatically, indicating parallel magnetization alignment. At $30 \mathrm{~K}$ in $0.2 \mathrm{mT}$ immediately after ZFC (Fig. 8c), we obtain a low value of $\beta$, corresponding to antiparallel alignment. After field cycling at $30 \mathrm{~K}$ (Fig. 8d), $\beta$ increases only slightly, but is still below unity, indicating that now the antiparallel alignment is very robust at this temperature. The PNR data thus indicate that the top and bottom layers spontaneously magnetize in the antiparallel (AF) configuration at both $5 \mathrm{~K}$ and $30 \mathrm{~K}$, thus confirming the presence of AF IEC. However, the cycling process shows the antiparallel state not to be robust at $5 \mathrm{~K}$, presumably due to a strong cubic anisotropy field at this low temperature, which locks the FM alignment after field cycling. However, the AF alignment clearly becomes robust at higher temperatures, as shown by the value of $\beta<1$ after cycling at $30 \mathrm{~K}$, as expected from magnetization measurements shown previously.

For completeness we also used PNR to measure the saturation magnetization of the layers at $810 \mathrm{mT}$ at $30 \mathrm{~K}$ and $40 \mathrm{~K}$, where the results were $M_{\text {top }}=+12 \mathrm{kA} \mathrm{m}^{-1}$, $M_{\text {bot }}=+24 \mathrm{kA} \mathrm{m}^{-1}$ and $M_{\mathrm{top}}=+11 \mathrm{kA} \mathrm{m}^{-1}$, $M_{\text {bot }}=+20 \mathrm{kA} \mathrm{m}^{-1}$, respectively, in order to check that the Curie temperatures of both the bottom and the top GaMnAs layers are above $40 \mathrm{~K}$. This conclusion is also
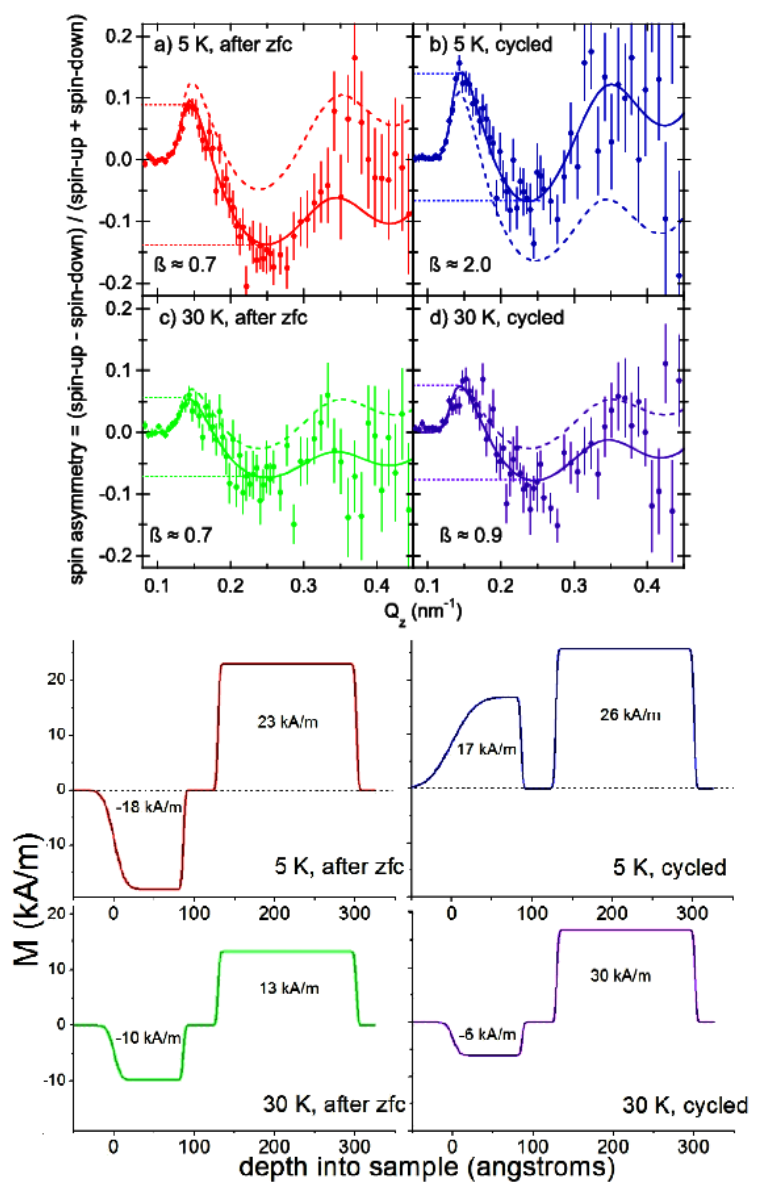

Fig. 8. Fitted PNR data of the trilayer sample plotted as $Q$-dependent spin asymmetry. Part (a) corresponds to spin-asymmetry after cooling in zero field to $5 \mathrm{~K}$, collected in an applied field of $0.65 \mathrm{mT}$; (b) shows ZFC data at $5 \mathrm{~K}$ after cycling and returning to $0.65 \mathrm{mT}$; (c) shows spin-asymmetry obtained in $0.18 \mathrm{mT}$ after cooling in zero field to $30 \mathrm{~K}$; and (d) shows spin asymmetry for the ZFC case obtained at $30 \mathrm{~K}$ after cycling and returning to $0.18 \mathrm{mT}$. The magnetic field was applied along the [110] direction. The solid lines are best fits to the data. Dashed lines are fits obtained with the same model, but with the magnetization of the top GaMnAs layer reversed. The magnetization profiles obtained from the best fits are plotted on the bottom.

supported by the SQUID data [24], which show that with an applied field of $50 \mathrm{mT}$ there is no dip in the magnetization curve. (A significant dip would be indicative of the two ferromagnetic layers having different Curie temperatures.)

\subsection{Magnetotransport observed on GaMnAs/GaAs/GaMnAs trilayers}

For magnetotransport measurements the trilayer was patterned into a Hall bar in the form of a rectangular strip $200 \mu \mathrm{m}$ long and $10 \mu \mathrm{m}$ wide, as shown in the inset in Fig. 9a, with gold wires attached to each terminal by indium contacts. The current through the Hall bar 
was along the [110] direction. The sample was mounted in a $4 \mathrm{~K}$ cryostat such that a magnetic field $\boldsymbol{H}$ could be applied in the plane of the sample. Magnetoresistance (MR) was measured at various azimuthal angles $\phi_{H}$ of the applied field (see inset in Fig. 9a). The characteristics of MR as a function of applied field $\boldsymbol{H}$ in thin GaMnAs films are well known [12, 27, 28]. In particular, the in-plane MR data typically shows a "two-horn" pattern, where the two kinks result from the switching of the direction of magnetization at the coercive field. This feature is observed in our trilayer at $4 \mathrm{~K}$, where the magnetization switching in both layers occurs simultaneously, as seen in Fig. 9a. Let us note, however, that at the beginning of the measurement (first sweep immediately following ZFC), the resistance is higher than at any later time. The high resistance seen in this first sweep is the result of the antiparallel alignment of magnetizations of the two GaMnAs layers after ZFC, i.e., before cycling [12, 29], consistent with the $5 \mathrm{~K}$ ZFC SQUID and with the neutron data obtained at $5 \mathrm{~K}$, shown previously in this paper. At $30 \mathrm{~K}$, however, the robust AF IEC which occurs at this temperature is manifested as a distinct upward jump in MR that appears before the applied field returns to zero after cycling, as seen in Fig. 9b. This increase in resistance due to a spontaneous reorientation of $M$ to the antiparallel alignment suggests the potential for device applications of such magnetic semiconductor structures.

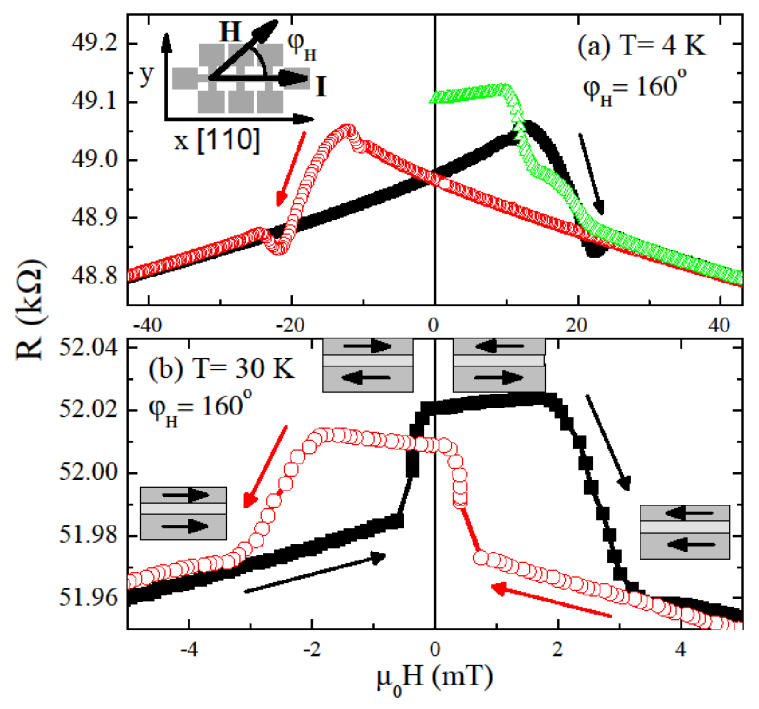

Fig. 9. (a) Field dependence of sheet resistance in a GaMnAs/GaAs/GaMnAs trilayer at $4 \mathrm{~K}$ after zero field cooling. (b) Field dependence of sheet resistance at $30 \mathrm{~K}$, after zero field cooling. Sketches show the configuration of the magnetizations at various fields in the resistance plot. The directions of the arrows are along the uniaxial easy axis [110] of GaMnAs. Inset in part (a) shows the Hall bar pattern, $\boldsymbol{H}$ indicates the applied magnetic field and $\boldsymbol{I}$ the current.

\section{Competition between IEC and magnetic anisotropy}

The PNR and $M(H)$ data which confirm the presence of AF IEC also enable us to better interpret the behavior of $M(T)$ in Fig. 5. This can be understood by considering the relative strengths of the coercive field associated with magnetocrystalline anisotropy and the interlayer exchange field $H_{\mathrm{E}}$ at different temperatures. It is well known that the cubic anisotropy field $H_{\mathrm{C}}$ decreases very rapidly with increasing temperature, while the uniaxial anisotropy field $H_{\mathrm{U}}$ decreases much more slowly. The temperature dependence of $H_{\mathrm{E}}$ is unknown, but based on our modeling of the free energy [24] we will argue that $H_{\mathrm{E}}$ falls off faster with increasing temperature than $H_{\mathrm{U}}$, and more slowly than $H_{\mathrm{C}}$. Thus, based on our results we suggest that, as the temperature increases, the mechanism dominating the hysteresis loop changes from the cubic anisotropy field $H_{\mathrm{C}}$ to interlayer exchange $H_{\mathrm{E}}$, and finally to the uniaxial anisotropy field $H_{\mathrm{U}}$. As seen in Fig. 5 (top), at $\approx 22 \mathrm{~K}$ the FC magnetization undergoes a precipitous drop, consistent with a transition between dominance by $H_{\mathrm{C}}$ (where the coercive field is larger than $H_{\mathrm{E}}$ ) and IEC dominance (where the coercive field is smaller than $H_{\mathrm{E}}$ ). In our trilayer this dominance of $H_{\mathrm{E}}$ prevails in only a short temperature range, since by the time the sample reaches $30 \mathrm{~K}$, the effect of uniaxial anisotropy $H_{\mathrm{U}}$ appears to overcome the contributions of both $H_{\mathrm{E}}$ and $H_{\mathrm{C}}$, as discussed below.

We now comment on our inability to observe IEC at higher temperatures $(>33 \mathrm{~K})$. It has been shown previously that the magnetic properties of GaMnAs depend on the electronic properties (e.g., on doping) of the layer on which it is grown [30,31], and that the resulting increased hole concentration in GaMnAs can result in switching of the uniaxial easy axis by $90^{\circ}$, from [110] to [1-10], [32, 33]. Since in the present case the top layer of the sample was grown on a heavily Be-doped spacer, it is possible that above a certain temperature the top GaMnAs layer experiences a reorientation of magnetization perpendicular to that of the bottom layer. In this situation the magnetizations of the two GaMnAs layers are orthogonal, and the contribution of the top layer to the magnetization (which was seen to reduce the total ZFC magnetization in Fig. 5 at low temperatures due to AF coupling) now disappears. This would explain the increase in $M(T)$ just above $30 \mathrm{~K}$ seen in both $\mathrm{FC}$ and $\mathrm{ZFC}$ data in Fig. 5. One should note here that the differences between the low-temperature FC and ZFC SQUID magnetizations as well as their increase (of 15 to 20\%) just above $30 \mathrm{~K}$ are very close to the respective values of $M$ obtained by PNR (see Fig. 8), thus providing additional corroboration for the model used in fitting the PNR results.

\section{Concluding remarks}

Our experiment shows that the AFM IEC appears in a GaMnAs/GaAs:Be/GaMnAs trilayer with individual layer thicknesses of $30 \mathrm{ML} / 15 \mathrm{ML} / 60 \mathrm{ML}$ and GaMnAs/ 
GaAs:Be SL with layer thicknesses of $50 \mathrm{ML} / 25 \mathrm{ML}$. This is interesting, because recent theoretical results [6-8] suggests that such AFM IEC requires considerably thinner FM and spacer layers. Those results indicate that the IEC will become too weak when the combined magnetic/non-magnetic layer thickness exceeds about $10 \mathrm{ML}$ $(\approx 3 \mathrm{~nm})$ or so, which is in clear contradiction with our experimental results, which demonstrate the existence of strong AFM IEC for combined FM/non-FM thicknesses of $50 \mathrm{ML} / 25 \mathrm{ML}$. Furthermore, the observation of AFM IEC was recently reported in GaMnAs/GaAs:Be SL even with thickness up to $50 \mathrm{ML} / 50 \mathrm{ML}$ [22].

In summary, our experiments conclusively show the existence of AFM exchange coupling between magnetic GaMnAs layers in GaMnAs/GaAs/GaMnAs multilayer structures (superlattices and trilayers). This coupling depends on the thickness and the doping of the spacer layers. We have observed that the relative orientation of $M$ in the two layers is determined by the competition between an interlayer exchange coupling and magnetic anisotropy intrinsic to GaMnAs. In measurements carried out so far the AFM coupling is strong only in a narrow temperature range. However, based on our new understanding of the mechanisms that allow for robust AFM coupling in that range (including the role of magnetic anisotropy and its temperature dependence), we hope to significantly extend the range of AFM IEC in future experiments.

\section{Acknowledgments}

This work was supported by NSF Grant DMR10-05851; and by the National Research Foundation of Korea (NRF) grant funded by the Korea government (MEST) (No. 2010-0025880).

\section{References}

[1] H. Ohno, Science 281, 951 (1998).

[2] T. Jungwirth, Jairo Sinova, J. Masek, J. Kucera, A.H. MacDonald, Rev. Mod. Phys. 78, 809 (2006).

[3] L. Chen, S. Yan, P.F. Xu, L. Lu, W.Z. Wang, J.J. Deng, X. Qian, Y. Ji, J.H. Zhao, Appl. Phys. Lett. 95, 182505 (2009).

[4] S. Lee, J.H. Chung, X.Y. Liu, J.K. Furdyna, B.J. Kirby, Mater. Today 12, 14 (2009).

[5] T. Jungwirth, W.A. Atkinson, B.H. Lee, A.H. MacDonald, Phys. Rev. B 59, 9818 (1999).

[6] P. Sankowski, P. Kacman, Phys. Rev. B 71, 201303(R) (2005).

[7] A.D. Giddings, T. Jungwirth, B.L. Gallagher, Phys. Status Solidi C 3, 4070 (2006).

[8] K. Szalowski, T. Balcerzak, Phys. Rev. B 79, 214430 (2009).

[9] H. Kepa, G. Springholz, T.M. Giebultowicz, K.I. Goldman, C.F. Majkrzak, P. Kacman, J. Blinowski, S. Holl, H. Krenn, G. Bauer, Phys. Rev. B 68, 024419 (2003).

[10] B.J. Kirby, J.A. Borchers, X. Liu, Z. Ge, Y.J. Cho, M. Dobrowolska, J.K. Furdyna, Phys. Rev. B 76, 205316 (2007).
[11] N. Akiba, F. Matsukura, A. Shen, Y. Ohno, H. Ohno, A. Oiwa, S. Katsumoto, Y. Iye, Appl. Phys. Lett. $\mathbf{7 3}$ 2122 (1998).

[12] D. Chiba, N. Akiba, F. Matsukura, Y. Ohno, H. Ohno, Appl. Phys. Lett. 77, 1873 (2000).

[13] H. Kępa, J. Kutner-Pielaszek, A. Twardowski, C.F. Majkrzak, J. Sadowski, T. Story, T.M. Giebultowicz, Phys. Rev. B 64, 121302(R) (2001).

[14] W. Szuszkiewicz, E. Dynowska, B. Hennion, F. Ott, M. Jouanne, J.F. Morhange, Acta Phys. Pol. A 100, 335 (2001d)

[15] S.J. Chung, S. Lee, I.W. Park, X. Liu, J.K. Furdyna, J. Appl. Phys. 95, 7402 (2004).

[16] P.A. Kienzle, K.V. O’Donovan, J.F. Ankner, N.F. Berk, C.F. Majkrzak, http://www.ncnr.nist.gov/ reflpak, 2000-2006.

[17] D.V. Baxter, D. Ruzmetov, J. Scherschligt, Y. Sasaki, X. Liu, J.K. Furdyna, C.H. Mielke, Phys. Rev. B $6 \mathbf{6 5}$ 212407 (2002).

[18] K.Y. Wang, K.W. Edmonds, R.P. Campion, L.X. Zhao, C.T. Foxon, B.L. Gallagher, Phys. Rev. B 72, 085201 (2005).

[19] J.H. Chung, S.J. Chung, S. Lee, B.J. Kirby, J.A. Borchers, Y.J. Cho, X. Liu, J.K. Furdyna, Phys. Rev. Lett. 101, 237202 (2008).

[20] M.N. Baibich, J.M. Broto, A. Fert, F.N. Van Dau, F. Petroff, P. Etienne, G. Creuzet, A. Friederich, J. Chazelas, Phys. Rev. Lett. 61, 2472 (1988).

[21] G. Binasch, P. Grunberg, F. Saurenbach, W. Zinn, Phys. Rev. B 39, 4828 (1989).

[22] S. Chung, S. Lee, J.-H. Chung, T. Yoo, H. Lee, B. Kirby, X. Liu, J. Furdyna, Phys. Rev. B 82, 054420 (2010).

[23] U. Welp, V.K. Vlasko-Vlasov, X. Liu, J.K. Furdyna, T. Wojtowicz, Phys. Rev. Lett. 90, 167206 (2003).

[24] J. Leiner, K. Tivakornsasithorn, X. Liu, J.K. Furdyna, M. Dobrowolska, B.J. Kirby, H. Lee, T. Yoo, S. Lee, J. Appl. Phys. 109, 07C307 (2011).

[25] C.F. Majkrzak, Physica B 221B, 342 (1996).

[26] C.F. Majkrzak, in: Neutron Scattering from Magnetic Materials, Ed. T. Chatterji, Elsevier Science, New York 2005, p. 397.

[27] H.X. Tang, R.K. Kawakami, D.D. Awschalom, M.L. Roukes, Phys. Rev. Lett. 90, 107201 (2003).

[28] M.H. Jung, S. Park, J. Eom, S.H. Chun, K. Shin, J. Appl. Phys. 104, 083908 (2008).

[29] M. Tanaka, Y. Higo, Phys. Rev. Lett. 87, 026602 (2001).

[30] T. Wojtowicz, W.L. Lim, X. Liu, M. Dobrowolska, J.K. Furdyna, K.M. Yu, W. Walukiewicz, Appl. Phys. Lett. 83, 4220 (2003).

[31] J.K. Furdyna, T. Wojtowicz, X. Liu, K.M. Yu, W. Walukiewicz, I. Vurgaftman, J.R. Meyer, J. Phys., Condens. Matter 16, S5499 (2004).

[32] M. Sawicki, K.Y. Wang, K.W. Edmonds, R.P. Campion, C.R. Staddon, N.R.S. Farley, C.T. Foxon, E. Papis, E. Kaminska, A. Piotrowska, T. Dietl, B.L. Gallagher, Phys. Rev. B 71, 121302(R) (2005).

[33] S. Chung, H.C. Kim, S. Lee, X. Liu, J.K. Furdyna, Solid State Commun. 149, 1739 (2009). 